Article

\title{
Bioassay-Guided Separation of Centipeda minima Using Comprehensive Linear Gradient Centrifugal Partition Chromatography
}

\author{
Ji Hoon Kim ${ }^{1}$, Eun Ju Jung ${ }^{1}$, Yun Jung Lee ${ }^{1}$, En Mei Gao ${ }^{1}$, Ahmed Shah Syed ${ }^{2}$ \\ and Chul Young Kim 1,*(D) \\ 1 College of Pharmacy and Institute of Pharmaceutical Science and Technology, Hanyang University, Ansan, \\ Gyeonggi-do 15588, Korea; gg890718@gmail.com (J.H.K.); jejs2@naver.com (E.J.J.); \\ sopihya@naver.com (Y.J.L.); rhdmsal@hanyang.ac.kr (E.M.G.) \\ 2 Department of Pharmacognosy, Faculty of Pharmacy, University of Sindh, Jamshoro 76088, Pakistan; \\ shahahmed454@gmail.com \\ * Correspondence: chulykim@hanyang.ac.kr; Tel.: +82-31-400-5809; Fax: +82-31-400-5958
}

Received: 8 June 2020; Accepted: 2 July 2020; Published: 6 July 2020

\begin{abstract}
A comprehensive linear gradient solvent system for centrifugal partition chromatography (CPC) was developed for the bioassay-guided isolation of natural compounds. The gradient solvent system consisted of three different ternary biphasic solvents types: $n$-hexane-acetonitrile-water (10:2:8, $v / v)$, ethyl acetate-acetonitrile-water $(10: 2: 8, v / v)$, and water-saturated $n$-butanol-acetonitrile-water $(10: 2: 8, v / v)$. The lower phase of the $n$-hexane-acetonitrile-water $(10: 2: 8, v / v)$ was used as the stationary phase, while its upper phase, as well as ethyl acetate-acetonitrile-water (10:2:8), and water-saturated $n$-butanol-acetonitrile-water $(10: 2: 8, v / v)$ were pumped to generate a linear gradient elution, increasing the mobile phase polarity. We used the gradient CPC to identify antioxidant response elements (AREs), inducing compounds from Centipeda minima, using an ARE-luciferase assay in HepG2 cells, which led to the purification of the active molecules 3-methoxyquercetin and brevilin A. The developed CPC solvent systems allow the separation and isolation of compounds with a wide polarity range, allowing active molecule identification in the complex crude extract of natural products.
\end{abstract}

Keywords: centrifugal partition chromatography; comprehensive linear gradient elution; bioactivity-guided isolation; 3-methoxyquercetin; brevilin A; Centipeda minima

\section{Introduction}

For centuries, medicinal plants have been valuable sources of functional ingredients, playing an important role in health maintenance, as well as disease prevention and treatment. Natural medicinal plant products provide unlimited opportunities for nutraceuticals, functional foods, natural health products, and new drug leads due to the considerable chemical diversity they make available [1-3]. In order to identify bioactive compounds from natural extracts, bioassay-guided fractionation is a common approach, performed through the following process: (i) solvent extraction from crude materials, (ii) fractionation of the liquid-liquid extraction and bioassay, (iii) fractionation using open column chromatography or prep-HPLC and bioassay screening of each fraction, (iv) active molecule isolation from the active fractions, and (v) structural elucidation of the isolated molecules and the evaluation of their bioactivity [4]. However, loss of activity or failure of the bioactive compound isolation from crude extracts during the bioactivity-guided isolation are common problems. Generally, the degradation or irreversible adsorption in a solid matrix during the purification process are the main reasons for these concerns. In addition, natural products are complex mixtures containing multiple components, and the conventional bioassay-guided fractionation strategy is time- and 
resource-intensive. Therefore, a more practical method for herbal medicine-derived active component discovery and purification would be required [5]. In order to solve these problems, countercurrent separation could provide an excellent alternative, such as centrifugal partition chromatography (CPC) or high-speed counter-current chromatography (HSCCC). CPC is a type of support-free liquid-liquid partition chromatography, with a low risk of sample denaturation, as well as total sample recovery, and large load capacity that has been widely used for the separation of constituents with bioactivity [6-10]. Despite the above-mentioned advantages, the CPC-mediated bioactivity-guided separation has several complementary points: (i) most solvent systems are limited to separating constituents with a narrow polarity range, (ii) optimizing a biphasic solvent system is a time-consuming process, as the partition coefficient of each component of the two layers should be compared in different ratios of the various solvents using HPLC or TLC. For the CPC-mediated activity-guided separation, a gradient biphasic solvent system that extends an extreme polarity range would be required without calculating the $\mathrm{K}$ values [11-14].

Centipeda minima is a traditional medicine used for treating cancer, sinusitis, swelling, and rhinitis. Phytochemical studies have isolated chlorogenic acid, cryptochlorogenic acid, caffeic acid, rutin, isochlorogenic acid $\mathrm{B}$, kaempferol-3-O-rutinoside, isochlorogenic acid $\mathrm{A}$, isochlorogenic acid $\mathrm{C}$, 3-methoxyquercetin, brevilin $\mathrm{A}$, arnicolide $\mathrm{D}$, and arnicolide $C$ from $C$. minima $[15,16]$. A previous study reported on the antioxidant effect of $C$. minima through the activation of the nuclear factor erythroid 2-related factor 2 signaling pathway from [17]. Therefore, C. minima was selected as a model sample to identify the antioxidant response element (ARE)-inducing compounds using a comprehensive linear-gradient CPC.

This study aimed at developing a linear gradient solvent system for CPC in order to fulfill compounds with a wide polarity range for the screening and separation of active constituents from herbal medicines. Based on the conventional liquid-liquid extraction approach, $n$-hexane, ethyl acetate, and water-saturated $n$-butanol were chosen as less polar solvents in ternary biphasic solvent systems. Considering the settling time and upper/lower volume ratios, acetonitrile was used as the polarity modifier. The developed gradient solvent system was applied for the activity-guided purification with an ARE-luciferase reporter assay in HepG2 cells transfected with the C. minima ARE gene.

\section{Results and Discussion}

\subsection{Gradient Biphasic Solvent System Selection}

As described previously, C. minima extract (CME) consists of mixtures with a wide range of polarities that are well-represented by the various features of natural products (Figure S3). In order to separate these CMEs through bioassay-guiding, we developed a comprehensive linear gradient solvent system that covered a wide polarity range, from nonpolar to extremely polar, with no K value calculation necessity. As shown in Figure 1, the CME consisted of compounds with a wide polarity range, a comprehensive gradient CPC operation would thus be needed to recover all the introduced components. Since the gradient solvent systems should satisfy various polar compounds and stable stationary phases, the aqueous phase was used as the stationary phase and the organic phase was used as the mobile phase with increasing polarities. Then, the organic layer polarity was sequentially increased with $n$-hexane, ethyl acetate, and $n$-butanol. In addition, as $n$-butanol was less polar to achieve an appropriate partition coefficient for a very polar constituent, a ternary solvent system was introduced in order to separate a suitable polarity for very polar constituents [18,19].

As a result, gradient ternary biphasic solvent systems were divided into three polarity groups: nonpolar, intermediate, and polar conditions, consisting of $n$-hexane- $X$-water, ethyl acetate-X-water, and water-saturated $n$-butanol-X-water, respectively. $\mathrm{X}$ stands for the tested modifier solvents, which were methanol, ethanol, acetonitrile, or isopropanol in this study. The ternary biphasic solvent systems, phase ratio, and settling time are described in Table 1. According to the settling time and biphasic solvent ratio (Figure S1, Tables S1 and S2), acetonitrile was 
determined as a modifier solvent. Finally, a volume ratio of 10:2:8 of $n$-hexane-acetonitrile-water, ethyl acetate-acetonitrile-water, and water-saturated $n$-butanol-acetonitrile-water were selected as linear-gradient solvent systems. Thus, three ternary biphasic solvent systems were separately prepared, and the lower layer of $n$-hexane-acetonitrile-water $(10: 2: 8, v / v / v)$ was used as the stationary phase. Then, the upper layer of $n$-hexane-acetonitrile-water, ethyl acetate-acetonitrile-water, and water-saturated $n$-butanol-acetonitrile-water $(10: 2: 8, v / v / v)$ were pumped to generate a linear gradient elution, thereby increasing the mobile phase polarity to recover all CME compounds. In the case of isopropanol as a modifier solvent, the initial pressure was too high for the upper mobile phase to flow out in the actual CPC procedure.

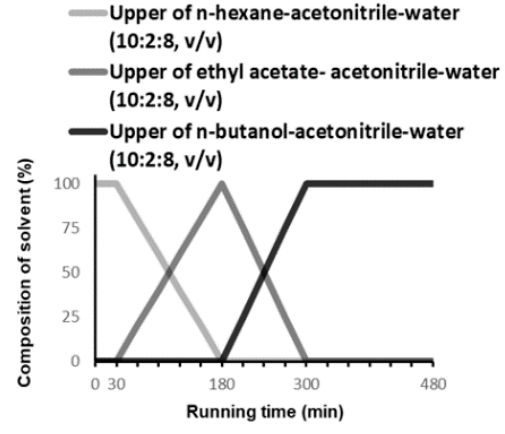

(a)

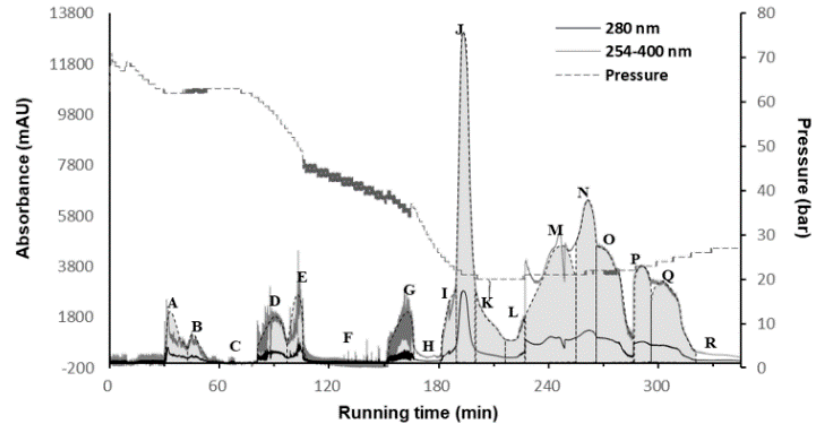

(b)

Figure 1. (a) Centrifugal partition chromatography (CPC) operation and chromatogram. Mobile phase composition with linear-gradient elution and (b) CPC separation chromatogram at $280 \mathrm{~nm}$ and scan mode: $254-320 \mathrm{~nm}$. The details are described in Section 3.6. CPC procedure.

Table 1. Phase ratios and settling times of each ternary biphasic solvent system.

\begin{tabular}{|c|c|c|c|c|c|c|c|c|c|c|c|}
\hline \multicolumn{4}{|c|}{ Less Polar Solvent Systems } & \multicolumn{4}{|c|}{ Medium Polar Solvent Systems } & \multicolumn{4}{|c|}{ Polar Solvent Systems } \\
\hline $\begin{array}{l}\text { Solvent } \\
\text { System }\end{array}$ & $\begin{array}{l}\text { Volume } \\
\text { Ratios } \\
(v / v / v)\end{array}$ & $\begin{array}{l}\text { Phase } \\
\text { Ratios }\end{array}$ & $\begin{array}{l}\text { Settling } \\
\text { Time }\end{array}$ & $\begin{array}{l}\text { Solvent } \\
\text { System }\end{array}$ & $\begin{array}{l}\text { Volume } \\
\text { Ratios } \\
(v / v / v)\end{array}$ & $\begin{array}{l}\text { Phase } \\
\text { Ratios }\end{array}$ & $\begin{array}{l}\text { Settling } \\
\text { Time }\end{array}$ & $\begin{array}{l}\text { Solvent } \\
\text { System }\end{array}$ & $\begin{array}{l}\text { Volume } \\
\text { Ratios } \\
(v / v / v)\end{array}$ & $\begin{array}{l}\text { Phase } \\
\text { Ratios }\end{array}$ & $\begin{array}{c}\text { Settling } \\
\text { Time }\end{array}$ \\
\hline \multirow{2}{*}{$\mathrm{H} / \mathrm{M} / \mathrm{W}$} & $10: 1: 9$ & $50 / 50$ & $12 \mathrm{~s}$ & \multirow{2}{*}{$\mathrm{EA} / \mathrm{M} / \mathrm{W}$} & $10: 1: 9$ & $58 / 42$ & $19 \mathrm{~s}$ & \multirow{2}{*}{$\mathrm{B} / \mathrm{M} / \mathrm{W}$} & $10: 1: 9$ & $50 / 50$ & $26 \mathrm{~s}$ \\
\hline & $10: 2: 8$ & $50 / 50$ & $12 \mathrm{~s}$ & & $10: 2: 8$ & $58 / 42$ & $20 \mathrm{~s}$ & & $10: 2: 8$ & $56 / 44$ & $3 \mathrm{~m} 17 \mathrm{~s}$ \\
\hline \multirow{2}{*}{$\mathrm{H} / \mathrm{E} / \mathrm{W}$} & 10:1:9 & $50 / 50$ & $>4 \mathrm{~m}$ & \multirow{2}{*}{$\mathrm{EA} / \mathrm{E} / \mathrm{W}$} & 10:1:9 & $48 / 52$ & $13 \mathrm{~s}$ & \multirow{2}{*}{$\mathrm{B} / \mathrm{E} / \mathrm{W}$} & 10:1:9 & $48 / 52$ & $1 \mathrm{~m} 30 \mathrm{~s}$ \\
\hline & $10: 2: 8$ & $49 / 51$ & $50 \mathrm{~s}$ & & $10: 2: 8$ & $52 / 48$ & $19 \mathrm{~s}$ & & $10: 2: 8$ & $57 / 43$ & $>4 \mathrm{~m}$ \\
\hline \multirow{4}{*}{$\mathrm{H} / \mathrm{I} / \mathrm{W}$} & $10: 1: 9$ & $50 / 50$ & $>4 \mathrm{~m}$ & \multirow{4}{*}{ EA/I/W } & 10:1:9 & $50 / 50$ & $14 \mathrm{~s}$ & \multirow{4}{*}{$\mathrm{B} / \mathrm{I} / \mathrm{W}$} & 10:1:9 & $50 / 50$ & $15 \mathrm{~s}$ \\
\hline & $10: 2: 8$ & $51 / 49$ & $24 \mathrm{~s}$ & & $10: 2: 8$ & $54 / 46$ & $19 \mathrm{~s}$ & & $10: 2: 8$ & $58 / 42$ & $24 \mathrm{~s}$ \\
\hline & $10: 3: 7$ & $52 / 48$ & $20 \mathrm{~s}$ & & $10: 3: 7$ & $62 / 38$ & $22 \mathrm{~s}$ & & 10:3:7 & $70 / 30$ & $37 \mathrm{~s}$ \\
\hline & $10: 4: 6$ & $54 / 46$ & $17 \mathrm{~s}$ & & $10: 4: 6$ & $74 / 26$ & $27 \mathrm{~s}$ & & $10: 4: 6$ & $96 / 4$ & $1 \mathrm{~m} 40 \mathrm{~s}$ \\
\hline \multirow{2}{*}{$\mathrm{H} / \mathrm{A} / \mathrm{W}$} & $10: 1: 9$ & $50 / 50$ & $14 \mathrm{~s}$ & \multirow{2}{*}{$\mathrm{EA} / \mathrm{A} / \mathrm{W}$} & 10:1:9 & $50 / 50$ & $10 \mathrm{~s}$ & \multirow{2}{*}{$\mathrm{B} / \mathrm{A} / \mathrm{W}$} & 10:1:9 & $50 / 50$ & $36 \mathrm{~s}$ \\
\hline & $10: 2: 8$ & $50 / 50$ & $11 \mathrm{~s}$ & & $10: 2: 8$ & $56 / 44$ & $11 \mathrm{~s}$ & & $10: 2: 8$ & $60 / 40$ & $43 \mathrm{~s}$ \\
\hline
\end{tabular}

H-n-hexane; M-methanol; W-water; E-ethanol; I-isopropanol; A-acetonitrile; EA-ethyl acetate; B-water-saturated $n$-butanol.

\subsection{Separation of the CPC Gradient Elution and ARE Induction Activity}

CPC was operated in a linear gradient of three ternary solvent systems, as shown in Figure 1a. The upper phase of the three ternary solvent systems was introduced sequentially as the mobile phase. The mobile phase consisted of $n$-hexane-acetonitrile-water $(10: 2: 8, v / v / v)$, ethyl acetate-acetonitrile-water (10:2:8, $v / v / v)$, and water-saturated $n$-butanol-acetonitrile-water (10:2:8).

As shown in Figure 1b, eighteen high-resolution fractions (A-R) were obtained, and the overall CPC operating time was $360 \mathrm{~min}$. The initial operating pressure was approximately $70 \mathrm{bar}$, then it began to decline after $80 \mathrm{~min}$, and was then maintained at 25 bar for approximately $180 \mathrm{~min}$. 
According to the CPC chromatogram, eighteen fractions were collected and evaporated to dryness: A (30-42.5 min, $173.7 \mathrm{mg}), \mathbf{B}$ (42.5-55 min, $44.8 \mathrm{mg}), \mathbf{C}$ (55-80 min, $48.6 \mathrm{mg}), \mathbf{D}$ (80-97.5 min, $76.7 \mathrm{mg})$, E (97.5-110 min, $119.2 \mathrm{mg}), \mathbf{F}(110-150 \mathrm{~min}, 51.5 \mathrm{mg}), \mathbf{G}(150-167 \mathrm{~min}, 65.4 \mathrm{mg}), \mathbf{H}$ (167-180.5 min, $25.7 \mathrm{mg}), \mathbf{I}(180.5-189 \mathrm{~min}, 55.6 \mathrm{mg}), \mathbf{J}(189-200.5 \mathrm{~min}, 100.8 \mathrm{mg}), \mathbf{K}(200.5-217.5 \mathrm{~min}, 106.7 \mathrm{mg})$, L (217.5-227 min, 75.8 mg), M (227-255.5 min, 243.9 mg), N (255.5-267 min, 116.6 mg), O (267-285.5 min, 97.2 $\mathrm{mg}$ ), $\mathbf{P}$ (285.5-295.5 $\mathrm{min}, 33.8 \mathrm{mg}), \mathbf{Q}$ (295.5-320.5 $\mathrm{min}, 82.4 \mathrm{mg}$ ), and $\mathbf{R}$ (320.5-residue in rotor, $2387.5 \mathrm{mg}$ ). After the CPC operation, the total sum of each fraction was $3905.9 \mathrm{mg}$ when $4.0 \mathrm{~g}$ of crude sample was loaded. The recovery rate was $97.6 \%$ (Table 2 ).

Table 2. Fraction weights and calculated concentrations.

\begin{tabular}{cccccccccc}
\hline Fraction & A & B & C & D & E & F & G & H & I \\
\hline Weight $(\mathrm{mg})$ & 173.7 & 44.8 & 48.6 & 76.7 & 119.2 & 51.5 & 65.4 & 25.7 & 55.6 \\
Weight ratio $(\%)$ & 4.4 & 1.1 & 1.2 & 2 & 3.1 & 1.3 & 1.7 & 0.7 & 1.4 \\
Calculated conc. $(\mu \mathrm{g} / \mathrm{mL})$ & 1.33 & 0.34 & 0.37 & 0.59 & 0.92 & 0.4 & 0.5 & 0.2 & 0.43 \\
\hline Fraction & $\mathbf{J}$ & $\mathbf{K}$ & $\mathbf{L}$ & $\mathbf{M}$ & $\mathbf{N}$ & $\mathbf{O}$ & $\mathbf{P}$ & $\mathbf{Q}$ & $\mathbf{R}$ \\
\hline Weight $(\mathrm{mg})$ & 100.8 & 106.7 & 75.8 & 243.9 & 116.6 & 97.2 & 33.8 & 82.4 & 2387.5 \\
Weight ratio $(\%)$ & 2.6 & 2.7 & 1.9 & 6.2 & 3 & 2.5 & 0.9 & 2.1 & 61.1 \\
Calculated conc. $(\mu \mathrm{g} / \mathrm{mL})$ & 0.77 & 0.82 & 0.58 & 1.87 & 0.9 & 0.75 & 0.26 & 0.63 & 18.34 \\
\hline
\end{tabular}

The fractions were profiled using HPLC/UV at $254 \mathrm{~nm}$, and we identified major peaks associated with the CME (fractions D, G, J, K, M, and $\mathbf{N}$ ) as showed in Figure 2. We next evaluated the CME for the ARE-inducing activity at a concentration of $30 \mu \mathrm{g} / \mathrm{mL}$, and 18 fractions were evaluated at the concentration applied at each assigned weight ratio (Table 2). The results showed that fraction $\mathbf{E}$ exerted the highest activity, followed by fractions D, A, and G. However, the activity of fraction A is not representative due to the following reasons: (i) it was treated at relatively high concentrations, (ii) the fraction chromatogram profiling shows the peaks of several substances, and (iii) compared with fraction $\mathbf{B}$, the chromatogram profiling showed a similar activity that was proportional with the concentration (treated fractions A and $\mathbf{B}$ with concentrations of 1.33 and $0.34, \mathbf{A}$ and $\mathbf{B}$ activities $=7.6-$ and 2.6-fold, respectively). Outside of that, fraction $\mathbf{I}-\mathbf{R}$ are non-active. Overall, the fractions including compound $\mathbf{1}$ and $\mathbf{2}$ in the HPLC chromatograms of fraction A-R were evaluated to be ARE-inducing activity. Therefore, fractions $\mathbf{D}-\mathbf{E}$ and $\mathbf{G}$ were further purified using preparative HPLC in order to obtain pure 3-methoxyquercetin (1) and brevilin A (2), respectively. The chemical structures of these compounds are shown in Figure 3. The physicochemical information of these compounds is indicated in Section structural information of the supplementary.

The ARE induction activity of the isolated compounds from active fractions D-E and G was assessed using a luciferase assay in HepG2 cells at serial concentrations of $0.5,1.5$, and $2.0 \mu \mathrm{M}$. The results showed that 3-methoxyquercetin (1) and brevilin A (2) enhanced the ARE activity in a dose-dependent manner (Figure 4). The 3-methoxyquercetin (1)-derived ARE-inducing activity was prominent. Significant antioxidant and hepatoprotective activities have already been reportedly attributed to 3-methoxyquercetin indicated by its ability to prevent liver cell death during $\mathrm{CCl}_{4}$ intoxication [20]. 
Chromatogram of CME and CPC fractions (UV at $254 \mathrm{~nm}$ )

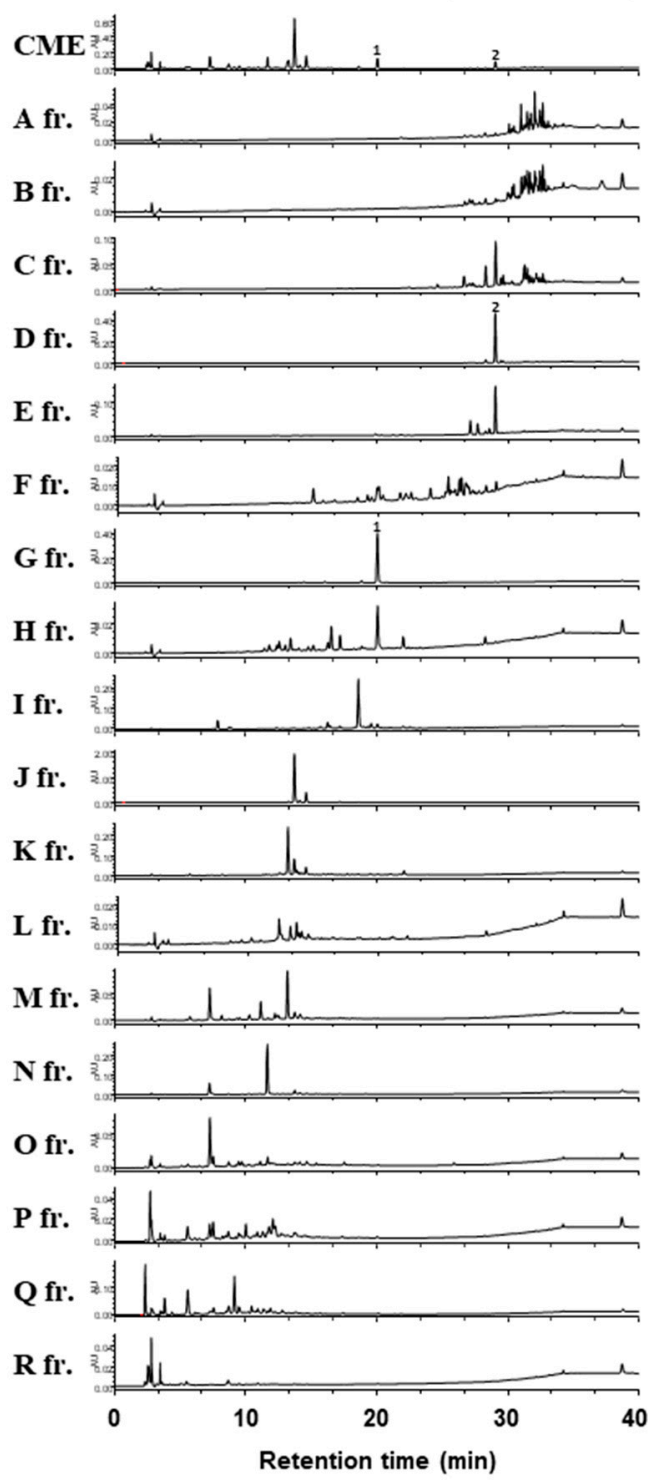

Luciferase reporter assay in HepG2 cells (vs. control)

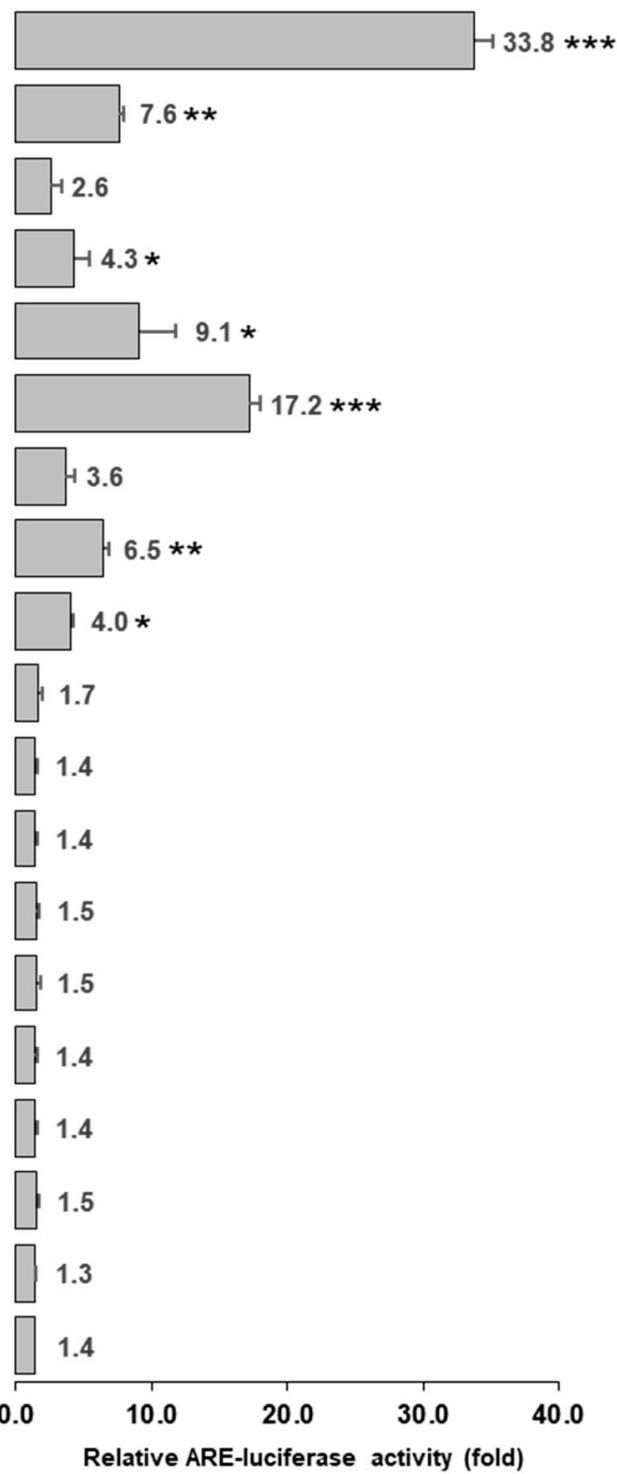

Figure 2. HPLC chromatograms and the relative antioxidant response element (ARE)-luciferase activities of the CPC-fractions (A-R). Each CPC fraction was analyzed using HPLC and the ARE induction activities were evaluated in ARE-HepG2 cells at concentrations applied at each assigned weight ratio (based on $30 \mu \mathrm{g} / \mathrm{mL}$ C. minima extract (CME)). Data are presented as the mean \pm S.E. $(n=3)$. ${ }^{*} p<0.05 ;{ }^{* *} p<0.01 ;{ }^{* * *} p<0.005$ (compared with the vehicle-treated control).<smiles>COc1cc(-c2oc3cc(O)cc(O)c3c(=O)c2O)ccc1O</smiles>

3-methoxyquercetin (1)<smiles>C/C=C(/C)C(=O)O[C@H]1C(C)C(=O)O[C@H]2C[C@@H](C)[C@H]3C=CC(=O)[C@@]3(C)[C@@H]21</smiles>

brevilin A (2)

Figure 3. Purified compound identification. The chemical structures of compounds $\mathbf{1}$ and $\mathbf{2}$. 


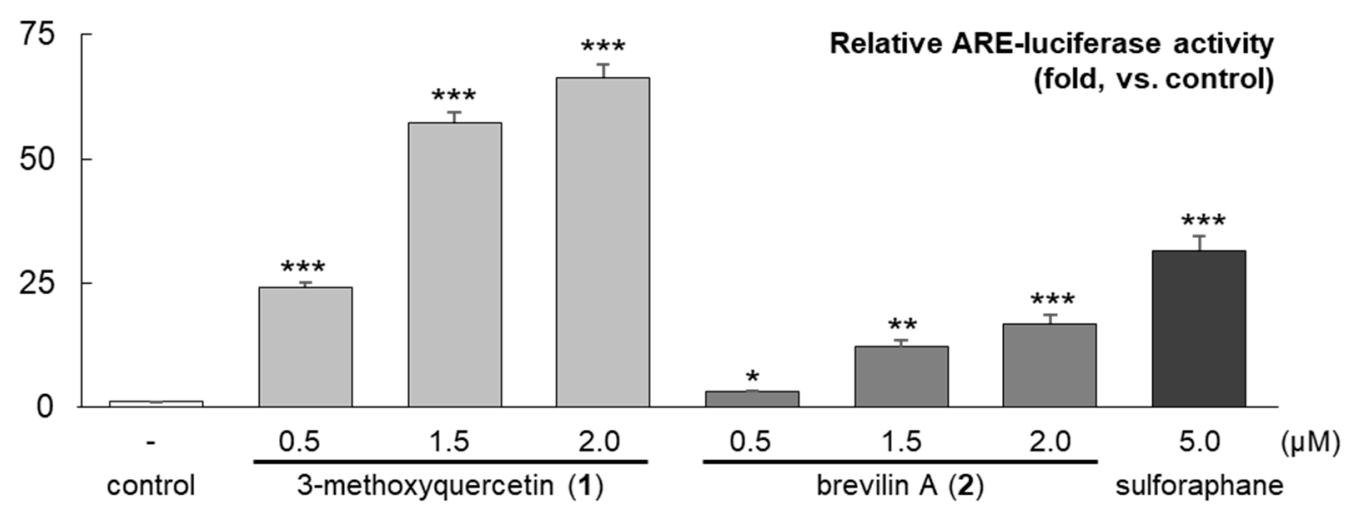

Figure 4. Relative ARE-luciferase activities of the purified compound. The ARE induction activities of compounds 1 and 2 were evaluated in ARE-HepG2 cells at concentrations of 0.5, 1.5, and $2.0 \mu \mathrm{M}$. Data are presented as the mean \pm S.E. $(n=3)$. ${ }^{*} p<0.05$; ${ }^{* *} p<0.01 ;{ }^{* * *} p<0.005$ (compared with the vehicle-treated control).

\section{Materials and Methods}

\subsection{Reagents and Materials}

All solvents used for the CPC were of analytical grade and purchased from Daejung Chemical (Gyeonggi-do, Republic of Korea). The HPLC-grade solvents were obtained from Fisher Scientific (Pittsburgh, PA, USA). The C. minima sample was purchased from the Kyungdong Oriental Herbal Market, Seoul, the Republic of Korea, in May 2017. A voucher specimen (HYUP-CM-001) was deposited in the Herbarium of the College of Pharmacy, Hanyang University.

\subsection{C. minima Extract Preparation}

The dried C. minima was chopped to the appropriate size, extracted by reflux with methanol (three times for $2 \mathrm{~h}$ each), and the extract solution was absorbed using Diaion HP-20 resin for chlorophyll removal followed by lyophilization in order to obtain a 4.31\%-yield dried extract (CME). The CME was stored at $-20^{\circ} \mathrm{C}$ until further use. For the cell treatment, the CME was dissolved in DMSO.

\subsection{Ternary Biphasic Solvent System Preparation}

In order to find a gradient elution solvent system capable of separating compounds with a wide polarity range, common solvents used for liquid-liquid extraction ( $n$-hexane, ethyl acetate, water-saturated $n$-butanol, and distilled water) were fixed, and the polarity modifier solvents (methanol, ethanol, acetonitrile, and isopropanol) were tested. The various ternary biphasic solvent systems are listed in Table 1.

\subsection{Settling Time and Phase Ratio Measurement}

The settling time, which highly correlated with the retention of the stationary phase, was expressed as the time to form a clear layer between the two phases $(1: 1, v / v)$ when mixed. The phase ratio was calculated as the ratio of each phase after mixing the upper phase with the lower phase.

\subsection{HPLC Analysis}

CME and CPC peak fractions were analyzed using a Waters Alliance 2695 HPLC system coupled with a Waters 2996 PDA detector (Waters, Milford, MA, USA) with a Capcellpak UG120 C18 column $(4.6 \times 250 \mathrm{~mm}, 5 \mu \mathrm{m}$, Shiseido, Tokyo, Japan). Acetonitrile $(0.1 \%$ formic acid, solvent A) and water $(0.1 \%$ formic acid, solvent B) were eluted in a gradient mode: $0-20 \mathrm{~min}, 10-40 \% \mathrm{~A} ; 20-25 \mathrm{~min}, 40-55 \%$ A; 25-30 min, 55-95\% A; and $40 \mathrm{~min}, 95 \%$ A. The flow rate was $1 \mathrm{~mL} / \mathrm{min}$, and the injection volume 
was $10 \mu \mathrm{L}$. The diode array detector measured the UV spectrum over a range of 200-450 $\mathrm{nm}$, and the chromatogram of the effluents was recorded at $254 \mathrm{~nm}$. The system was controlled using Waters Empower 2 Chromatography Software.

\subsection{CPC Procedure}

An SCPC-1000 (Armen instrument, St-Ave, France) apparatus and a Spot Prep II HPLC instrument were combined to form a CPC system. The preparation of the biphasic solvent system for the linear gradient elution mode was designed as follows: the upper and lower phase solvents were prepared when the solvents were perfectly mixed and completely equilibrated. The $1000 \mathrm{~mL}$ volume of the CPC rotor was filled with a lower layer of $n$-hexane-acetonitrile-water $(10: 2: 8, v / v / v)$ as the stationary phase at $50 \mathrm{~mL} / \mathrm{min}$ in ascending mode at a speed of $500 \mathrm{rpm}$. Then, the rotation speed of the rotor was accelerated to $800 \mathrm{rpm}$, and the upper layer as the mobile phase was carried into the rotor in descending mode at a flow rate of $10 \mathrm{~mL} / \mathrm{min}$. The CPC rotor reached equilibrium at the immiscible biphasic state $(320 \mathrm{~mL}$ of the stationary phase out of $1000 \mathrm{~mL}$ rotor volume, $69 \mathrm{bar})$. The elution was carried out in gradient mode with a mixed mobile phase of A (upper layer of $n$-hexane-acetonitrile-water, 10:2:8, v/v/v), B (upper layer of ethyl acetate-acetonitrile-water, 10:2:8, v/v/v), and C (upper layer of water-saturated $n$-butanol-acetonitrile-water, 10:2:8, v/v/v) with a flow rate of $10 \mathrm{~mL} / \mathrm{min}$ for 0-30 min (100\% A), 30-180 min (100\% A-100\% B), 180-300 $\min (100 \%$ B-100\% C), and 300-480 $\min (100 \%$ C). Thereafter, methanol was pumped for washing at $50 \mathrm{~mL} / \mathrm{min}$, and all the remaining samples were recovered. The CME (4.0 g) was dissolved in $20 \mathrm{~mL}$ of mixed upper and lower phases and subjected to the CPC system. The system was controlled using Armen Glider CPC V5.0b.09 Software.

\subsection{Cell Culture and Viability Assay}

The construction of the HepG2-ARE cells (transfected Pg14.37 [luc2P/ARE/ Hygro] (Promega)) was performed as described previously [21]. The HepG2-ARE cells were cultured in DMEM high glucose media (Hyclone, Logan, UT, USA) supplemented with 10\% FBS (Hyclone, Logan, UT), $1 \%$ penicillin-streptomycin (Hyclone, Logan, UT, USA), and 1\% hygromycin B (Invitrogen, Carlsbad, CA, USA). The cell viability was examined using a 3-(4,5-dimethylthiazol-2-yl)-2,5-diphenyl tetrazolium bromide (MTT) assay. HepG2 cells stably transfected with pGL4.37 (HepG2-ARE cells) were seeded at a density of $1 \times 10^{5}$ cells/well in 24-well plates for $24 \mathrm{~h}$. After serum starvation for $12 \mathrm{~h}$ and upon reaching an approximately $80 \%$ confluency, the cells were incubated with the major compounds for $24 \mathrm{~h}$. The cells were treated with $50 \mu \mathrm{L}$ MTT for $1 \mathrm{~h}$. The formazan precipitate was dissolved in $1 \mathrm{~mL}$ of dimethyl sulfoxide (DMSO), and the absorbance was measured at $570 \mathrm{~nm}$ using a microplate reader.

\subsection{ARE-Inducing Activity Assay}

HepG2-ARE cells were seeded at a density of $1 \times 10^{5}$ cells/well in 24 -well plates for $24 \mathrm{~h}$. The cells were starved for $12 \mathrm{~h}$ when they grew to approximately $80 \%$ of confluency and exposed to crude extract, CPC fractions, and purified compounds for an additional 24 . Then, the cells were lysed using $120 \mu \mathrm{L}$ of passive lysis buffer (Promega, Madison, WI, USA) in an ice rack and transferred into 1.5-mL tubes. The tubes were centrifuged at $1000 \mathrm{rpm}$ for $3 \mathrm{~min}$. Each supernatant $(30 \mu \mathrm{L})$ in the centrifuged tube was reacted with $60 \mu \mathrm{L}$ of assay substrate in a white 96-well plate. Finally, the luminescence was measured using an EnSpire multimode plate reader (PerkinElmer, Waltham, MA, USA). DMSO (below $0.1 \%$ ) was used as a vehicle, which was the negative control. Sulforaphane was used as a positive control.

\subsection{Protein Assay}

The protein was determined using the Pierce Micro BCA protein assay kit with BSA as a standard (Pierce No. 23227; Thermo Fisher Scientific, Illinois, USA). Each standard and unknown sample lysate $(10 \mu \mathrm{L})$ was replicated in a 96-well microplate. The working reagent $(200 \mu \mathrm{L})$ was added to each well. The BCA reagents A and B were mixed in a 49:1 volume ratio. After 30 min of incubation at 
$37^{\circ} \mathrm{C}$, the plate was cooled at room temperature and the absorbance was measured at $562 \mathrm{~nm}$ using a microplate reader. The calculated value of the protein assay was used as a factor to correct for ARE-inducing activity.

\subsection{Statistical Analysis}

All data are presented as the mean \pm standard error (S.E.) and the difference between the control and treatment groups was assessed using the Student's t-test. ${ }^{*} p<0.05,{ }^{* *} p<0.01,{ }^{* * *} p<0.005$ were considered statistically significant.

\section{Conclusions}

In this study, a comprehensive CPC gradient solvent system was developed for bioassay-guided isolation from the CME. The upper phase of three ternary solvent systems with $n$-hexane-acetonitrile-water, ethyl acetate-acetonitrile-water, and water-saturated $n$-butanol-acetonitrile-water (each 10:2:8, v/v/v) eluted in a linear gradient mode, and the ARE-inducing molecule 3-methoxyquercetin (1) and brevilin A (2) were purified from the CME. The overall results of this study demonstrate that a linear gradient CPC with three ternary solvent systems is suitable for screening bioactive molecules from natural products, even when the compounds have a wide polarity range.

Supplementary Materials: The following are available online at http://www.mdpi.com/1420-3049/25/13/3077/s1, Figure S1: Preparation of simulating solvent systems for the gradient elution, Figure S2: CME ARE-luciferase induction activity, Figure S3: CME and marker compound HPLC chromatograms. Table S1: Volume ratio and settling time of biphasic solvent systems, Table S2: The solvent composition in a simulating solvent system of $n$-hexane-acetonitrile-water, ethyl acetate-acetonitrile-water, and water-saturated $n$-butanol-acetonitrile-water $(10: 2: 8, v / v / v)$.

Author Contributions: Conceptualization, J.H.K. and C.Y.K.; Data curation, J.H.K. and E.J.J.; Formal analysis, J.H.K., E.J.J., Y.J.L., and E.M.G.; Funding acquisition, C.Y.K.; Investigation, J.H.K. and E.J.J.; Methodology, J.H.K.; Project administration, C.Y.K.; Resources, E.J.J.; Software, J.H.K. and A.S.S.; Supervision, C.Y.K.; Validation, C.Y.K.; Visualization, J.H.K.; Writing—original draft, J.H.K.; Writing—review \& editing, J.H.K. and C.Y.K. All authors have read and agreed to the published version of the manuscript.

Funding: This research was supported by the Basic Science Research Program through the National Research Foundation of Korea (NRF) funded by the Ministry of Science and ICT (NRF-2020R1A2C1009455 and NRF-2016R1A2B4014598).

Conflicts of Interest: The authors declare no conflicts of interest.

\section{References}

1. Tsao, R.; Deng, Z. Separation procedures for naturally occurring antioxidant phytochemicals. J. Chromatogr. B 2004, 812, 85-99. [CrossRef]

2. Atanasov, A.G.; Waltenberger, B.; Pferschy-Wenzig, E.M.; Linder, T.; Wawrosch, C.; Uhrin, P.; Temml, V.; Wang, L.; Schwaiger, S.; Heiss, E.H.; et al. Discovery and resupply of pharmacologically active plant-derived natural products: A review. Biotechnol. Adv. 2015, 33, 1582-1614. [CrossRef] [PubMed]

3. Bernardini, S.; Tiezzi, A.; Laghezza Masci, V.; Ovidi, E. Natural products for human health: An historical overview of the drug discovery approaches. Nat. Prod. Res. 2018, 32, 1926-1950. [CrossRef] [PubMed]

4. Nothias, L.F.; Nothias-Esposito, M.; da Silva, R.; Wang, M.; Protsyuk, I.; Zhang, Z.; Sarvepalli, A.; Leyssen, P.; Touboul, D.; Costa, J.; et al. Bioactivity-based molecular networking for the discovery of drug leads in natural product bioassay-guided fractionation. J. Nat. Prod. 2018, 81, 758-767. [CrossRef] [PubMed]

5. Kellogg, J.J.; Todd, D.A.; Egan, J.M.; Raja, H.A.; Oberlies, N.H.; Kvalheim, O.M.; Cech, N.B. Biochemometrics for natural products research: Comparison of data analysis approaches and application to identification of bioactive compounds. J. Nat. Prod. 2016, 79, 376-386. [CrossRef] [PubMed]

6. Alvi, K.A. Screening natural products: Bioassay-directed isolation of active components by dual-mode CCC. J. Liq. Chromatogr. Relat. Technol. 2001, 24, 1765-1773. [CrossRef]

7. Ignatova, S.; Sumner, N.; Colclough, N.; Sutherland, I. Gradient elution in counter-current chromatography: A new layout for an old path. J. Chromatogr. A 2011, 1218, 6053-6060. [CrossRef] 
8. Zhang, Y.Q.; Luo, J.G.; Han, C.; Xu, J.F.; Kong, L.Y. Bioassay-guided preparative separation of angiotensin-converting enzyme inhibitory $\mathrm{C}$-flavone glycosides from Desmodium styracifolium by recycling complexation high-speed counter-current chromatography. J. Pharm. Biomed. Anal. 2015, 102, $276-281$. [CrossRef]

9. Wang, M.; Gu, D.; Guo, X.; Li, H.; Wang, Y.; Guo, H.; Yang, Y.; Tian, J. Bioassay-guided isolation of an active compound with protein tyrosine phosphatase 1B inhibitory activity from Sargassum fusiforme by high-speed counter-current chromatography. J. Sep. Sci. 2016, 39, 4408-4414. [CrossRef]

10. Liu, M.; Liu, Q.; Chen, M.; Huang, X.; Chen, X. Large-scale separation of acetylcholinesterase inhibitors from Zanthoxylum nitidum by $\mathrm{pH}$-zone-refining counter-current chromatography target-guided by ultrafiltration high-performance liquid chromatography with ultraviolet and mass spectrometry screening. J. Sep. Sci. 2019, 42, 1194-1201. [CrossRef]

11. Zhang, Y.; Liu, C.; Zhang, Z.; Wang, J.; Wu, G.; Li, S. Comprehensive separation and identification of chemical constituents from Apocynum venetum leaves by high-performance counter-current chromatography and high performance liquid chromatography coupled with mass spectrometry. J. Chromatogr. B 2010, 878, 3149-3155. [CrossRef]

12. Ying, H.; Jiang, H.; Liu, H.; Chen, F.; Du, Q. Ethyl acetate- $n$-butanol gradient solvent system for high-speed countercurrent chromatography to screen bioactive substances in okra. J. Chromatogr. A 2014, 1359, 117-123. [CrossRef]

13. Leitão, G.G.; Costa, F.D.N. Gradient elution in countercurrent chromatography. Planta Med. 2015, 81, 1592-1596. [CrossRef]

14. Wang, Z.; Hwang, S.H.; Lim, S.S. Comprehensive profiling of minor tyrosinase inhibitors from Gastrodia elata using an off-line hyphenation of ultrafiltration, high-speed countercurrent chromatography, and high-performance liquid chromatography. J. Chromatogr. A 2017, 1529, 63-71. [CrossRef]

15. Chan, C.O.; Jin, D.P.; Dong, N.P.; Chen, S.B.; Mok, D.K.W. Qualitative and quantitative analysis of chemical constituents of Centipeda minima by HPLC-QTOF-MS \& HPLC-DAD. J. Pharm. Biomed. Anal. 2016, 125, 400-407.

16. Nguyen, N.Y.T.; Nguyen, T.H.; Dang, P.H.; Le Tran, Q. Three terpenoid glycosides of Centipeda minima. Phytochem. Lett. 2017, 21, 21-24. [CrossRef]

17. Wang, Y.J.; Wang, X.Y.; Hao, X.Y.; Yan, Y.M.; Hong, M.; Wei, S.F.; Zhou, Y.L.; Wang, Q.; Cheng, Y.X.; Liu, Y.Q. Ethanol Extract of Centipeda minima Exerts Antioxidant and Neuroprotective Effects via Activation of the Nrf2 Signaling Pathway. Oxid. Med. Cell. Longev. 2019, 2019, 1-16. [CrossRef]

18. Kim, C.Y.; Kim, J. Preparative isolation and purification of geniposide from gardenia fruits by centrifugal partition chromatography. Phytochem. Anal. 2007, 18, 115-117. [CrossRef]

19. Yeon, S.H.; Jeon, J.S.; Um, K.A.; Kim, C.Y.; Ahn, Y.J. Large-scale purification of unstable, water-soluble secologanic acid using centrifugal partition chromatography. Phytochem. Anal. 2018, 29, 487-492. [CrossRef]

20. Hubert, D.J.; Dawe, A.; Florence, N.T.; Gilbert, K.D.; Angele, T.N.; Buonocore, D.; Vita Finzi, P.; Vidari, G.; Bonaventure, N.T.; Marzatico, F.; et al. In vitro hepatoprotective and antioxidant activities of crude extract and isolated compounds from Ficus gnaphalocarpa. Inflammopharmacology 2011, 19, 35-43. [CrossRef]

21. Jeon, J.S.; Park, C.L.; Syed, A.S.; Kim, Y.M.; Cho, I.J.; Kim, C.Y. Preparative separation of sesamin and sesamolin from defatted sesame meal via centrifugal partition chromatography with consecutive sample injection. J. Chromatogr. B 2016, 1011, 108-113. [CrossRef]

Sample Availability: Samples of the 3-methoxyquercetin and brevilin A are available from the authors. 